${ }^{4}$ RCCS San Raffaele Hospital, Immunology, Rheumatology, Allergology and Rare Disease - Laboratory of Autoimmunity and Vascular Inflammation, Milan, Italy; ${ }^{5}$ ASST Gaetano Pini-Cto Institute, Division of Clinical Rheumatology Department of Clinical Sciences and Community Health, Research Center for Adult and Pediatric Rheumatic Diseases, Milan, Italy; ${ }^{6}$ School of Medicine, University of L'aquila, Department of Biotechnological and Applied Clinical Science, Rheumatology Unit, L'aquila, Italy; ${ }^{7}$ Istituto Auxologico Italiano, University of Milan, Department of Clinical Sciences and Community Health -; Experimental Laboratory of Immunorheumatological Researches, Milan, Italy; ${ }^{8}$ University of Rome Tor Vergata, Rheumatology, Allergology and Clinical Immunology, Department of "Medicina Dei Sistemi," Rome, Italy; ${ }^{9}$ Sapienza University of Rome, Reumatologia, Dipartimento di Scienze Cliniche, Internistiche, Anestesiologiche E Cardiovascolari, Rome, Italy; ${ }^{10}$ Polymedical Center for Prevention of Recurrent Spontaneous Abortion, Polymedical Center for Prevention of Recurrent Spontaneous Abortion, Rome, Italy; ${ }^{11}$ University Hospital of Padua, Rheumatology Unit, Department of Medicine, Padua, Italy; ${ }^{12}$ Ospedale Gaetano Pini, University of Milan, Division of Rheumatology, Department of Clinical Sciences and Community Health, Milan, Italy; ${ }^{13}$ Azienda Ospedaliera San Camillo-Forlanini, Rheumatology Unit, Roma, Italy; ${ }^{14}$ University of Pisa, Rheumatology Unit, Department of Clinical and Experimental Medicine, Pisa, Italy; ${ }^{15}$ University Of Ferrara, Division of Rheumatology, Department of Clinical Sciences, Ferrara, Italy; ${ }^{16}$ IRCCS San Raffaele Hospital - San Raffaele University, Unit of Immunology, Rheumatology, Allergy and Rare Diseases, Milan, Italy

Background: The role of complement in the antiphospholipid (aPL) related pathology has been widely studied in animal models. Antiphospholipid antibodies can induce fetal loss in experimental animals but mice deficient in specific complement components (C4, C3, C5) appear somehow protected. In addition, in pregnant mice injected with aPL, antibody deposition has been found at decidual level causing focal necrosis, apoptosis and neutrophil infiltrates and supporting aPL pathogenetic potential. On the other hand, human studies did find hypocomplementemia associated to pregnancy complications in patients with obstetric antiphospholipid syndrome (APS). These results, however, are not unanimously confirmed and, in addition, some studies only show increased levels of complement activation products (i.e. $\mathrm{Bb}$ ) and not decreased levels of $\mathrm{C} 3$ and/or C4. A recently study focusing on complement level in early pregnancy and before pregnancy showed a significant correlation with pregnancy complications and loss in a large cohort of primary APS.

Objectives: To investigate if the simple detection of low $\mathrm{C} 3$ and/or $\mathrm{C} 4$ could be considered a risk factor for adverse pregnancy outcome in APS and APL carriers pregnancies.

Methods: We performed a multicentric study including patients from 10 Italian and 1 Russian Centers. Data on pregnancies in women with primary APS $(n=434)$ and asymptomatic carriers with persistently positive aPL but not fulfilling clinical criteria for APS $(n=218)$ were retrospectively collected. Serum C3 and C4 levels were evaluated by nephelometry; hypocomplementemia was defined by local laboratory reference values. Statistical analysis was performed using GraphPad.

Results: Preconceptional complement levels and gestational outcome were available for 107 (25\%) pregnancies in APS out of 434 and for 196 (90\%) pregnancies in aPL carriers women out of 218. In pregnancies with low preconceptional C3 and/or C4, a significantly higher prevalence of pregnancy losses was observed $(p=0.019)$. A subgroup analysis focusing on triple aPL positive patients was also performed. Preconceptional low C3 and/or C4 levels were found to be associated with an increased rate of pregnancy loss $(p=0.027)$ in this subgroup also. Otherwise, adverse pregnancy outcomes in single or double aPL positive women were not related to preconception complement levels $(p=0.44)$ (Table 1$)$. Of note, all the pregnancy losses in the triple positive group occurred in patients treated with low dose aspirin and low molecular weight heparin from the time of positive pregnancy test.

Conclusion: Our findings confirm that decreased complement levels before pregnancy are associated with increased risk of adverse outcome. This has been seen only in in women with triple aPL positivity, indeed single or double positivity does not show this trend. Complement levels are cheap and easy to be measured therefore they could represent a useful aid to identify patients at increased risk of pregnancy loss. test positivity.

REFERENCES:

[1] De Carolis S, et al. Complementemia and obstetric outcome in pregnancy with antiphospholipid syndrome. Lupus (2012) 21:776-8

[2] Kim MY, et al. Complement activation predicts adverse pregnancy outcome in patients with systemic lupus erythematosus and/or antiphospholipid antibodies. Ann Rheum Dis (2018) 77:549-55.

[3] Fredi M, et al. Risk Factors for Adverse Maternal and Fetal Outcomes in Women With Confirmed aPL Positivity: Results From a Multicenter Study of 283 Pregnancies. Front Immunol. 2018 May 7;9:864.
Table 1.

\begin{tabular}{|c|c|c|c|c|c|c|}
\hline \multirow[b]{2}{*}{ Gestational outcome } & \multicolumn{2}{|c|}{$\begin{array}{l}\text { Triple aPL } \\
\text { positivity }\end{array}$} & \multicolumn{4}{|c|}{$\begin{array}{l}\text { Single or double aPL } \\
\text { positivity }\end{array}$} \\
\hline & $\begin{array}{l}\text { Low } \\
\text { C3/C4 } \\
(n=49)\end{array}$ & $\begin{array}{c}\text { Normal } \\
\text { C3/C4 } \\
(n=17)\end{array}$ & $\mathbf{p}$ & $\begin{array}{l}\text { Low } \mathrm{C}_{3} / \mathrm{C}_{4} \\
\quad(\mathrm{n}=57)\end{array}$ & $\begin{array}{c}\text { Normal } \\
\text { C3/C4 } \\
(n=165)\end{array}$ & $p$ \\
\hline Term live birth $(>37 w)$ & $15(31 \%)$ & $6(35 \%)$ & ns & $34(60 \%)$ & $110(67 \%)$ & ns \\
\hline Preterm live birth $(\leq 37 w)$ & $22(45 \%)$ & $11(65 \%)$ & ns & $15(26 \%)$ & $38(23 \%)$ & ns \\
\hline $\begin{array}{l}\text { Pregnancy losses (abortion } \\
\text { and miscarriages) }\end{array}$ & $12(24 \%)$ & $0(0 \%)$ & 0.027 & $8(14 \%)$ & $17(10 \%)$ & ns \\
\hline
\end{tabular}

Disclosure of Interests: None declared

DOI: 10.1136/annrheumdis-2021-eular.1824

\section{POS0738 \\ BLUNTED CEREBRAL OXYGENATION DURING EXERCISE IN NON-NEUROPSYCHIATRIC SYSTEMIC LUPUS ERYTHEMATOSUS}

N. Koletsos ${ }^{1}$, K. Dipla ${ }^{2}$, A. Triantafyllou ${ }^{1}$, A. Lazaridis ${ }^{1}$, N. Papadopoulos ${ }^{3}$, A. Zafeiridis ${ }^{2}$, S. Aslanidis ${ }^{4}$, S. Douma ${ }^{1}$, E. Gkaliagkousi ${ }^{1} .{ }^{1}$ Papageorgiou General Hospital, Aristotle University of Thessaloniki, ESH Hypertension Excellence Center, 3rd Department of Internal Medicine, Thessaloniki, Greece; ${ }^{2}$ Aristotle University of Thessaloniki, Exercise Physiology \& Biochemistry Laboratory, Department of Sport Sciences, Serres, Greece; ${ }^{3}$ Papageorgiou General Hospital, Rheumatology Department, Thessaloniki, Greece;

${ }^{4}$ Hippokration General Hospital, Rheumatology Department-2nd Propedeutic Department of Internal Medicine, Thessaloniki, Greece

Background: Systemic lupus erythematosus (SLE) is a chronic autoimmune disease affecting multiple organs, including the central nervous system. Subclinical brain lesions have been reported in SLE patients, even without overt neuropsychiatric manifestations (non-NPSLE). Studies using PET/MRI, examining structural or functional brain abnormalities in SLE, have been previously performed, either at rest or during a mental task (1-3). Exercise can be used to identify early alterations in brain oxygenation that might not detectable during resting conditions (4).

Objectives: Our study aimed to examine possible differences in cerebral oxygenation during a handgrip exercise test between SLE patients without neuropsychiatric manifestations and age-matched controls.

Methods: Fifty-two participants (26 non-NPSLE and 26 controls), following evaluation of handgrip strength, underwent a protocol involving a seated rest (baseline), a 3-min handgrip exercise (at $30 \%$ of maximal strength), and a 3-min recovery. Continuous-near-infrared-spectroscopy (NIRS) was used to monitor changes in cerebral-oxygenated hemoglobin $(\mathrm{O} 2 \mathrm{Hb})$, de-oxygenated $(\mathrm{HHb})$ and total-hemoglobin (tHb). Beat-by-beat blood pressure (Finapres) was continuously monitored.

Results: There were no differences between the two groups in age, body mass index, blood pressure, and smoking status. Median SLE duration was 7.5 (3.0 16.0) years. During exercise, cerebral $-\mathrm{O} 2 \mathrm{Hb}$ increased in both groups; however non-NPSLE exhibited a significantly lower increase in $\mathrm{O} 2 \mathrm{Hb}$ vs. controls (average response: $1.20 \pm 0.89$ vs. $2.33 \pm 1.61 \mu \mathrm{M}$, respectively, $\mathrm{p}<0.005$ ) and lower $\mathrm{tHb}$ responses $(\mathrm{p}<0.05)$, with no differences in $\mathrm{HHb}$

Conclusion: Our data show, for the first time, that SLE patients even without overt neuropsychiatric manifestations exhibit a blunted increase in cerebral-O2Hb during a submaximal exercise stimulus compared to age-matched controls. Examining brain oxygenation during a simple exercise task may assist in identifying patients with early alterations in cerebral function.

\section{REFERENCES:}

[1] Mak A, Ren T, Fu EH yun, Cheak AA cia, Ho RCM. A Prospective Functional MRI Study for Executive Function in Patients with Systemic Lupus Erythematosus Without Neuropsychiatric Symptoms. Semin Arthritis Rheum. 2012;41(6):849-58.

[2] Kozora E, Brown MS, Filley CM, Zhang L, Miller DE, West SG, et al. Memory impairment associated with neurometabolic abnormalities of the hippocampus in patients with non-neuropsychiatric systemic lupus erythematosus. Lupus. 2011;20(6):598-606.

[3] Mackay M, Vo A, Tang CC, Small M, Anderson EW, Ploran EJ, et al. Metabolic and microstructural alterations in the SLE brain correlate with cognitive impairment. JCI Insight. 2019;4(1).

[4] Triantafyllou GA, Dipla K, Triantafyllou A, Gkaliagkousi E, Douma S. Measurement and Changes in Cerebral Oxygenation and Blood Flow at Rest and During Exercise in Normotensive and Hypertensive Individuals. Vol. 22, Current Hypertension Reports. Springer; 2020.

Disclosure of Interests: None declared

DOI: 10.1136/annrheumdis-2021-eular.1867 